\title{
Dimensions of the Foot Muscles in the Lowland Gorilla
}

\author{
Motoharu OISHI ${ }^{1) *}$, Naomichi OGIHARA ${ }^{2)}$, Hideki ENDO ${ }^{3)}$, Teruyuki KOMIYA ${ }^{4)}$, Shin-ichiro KAWADA ${ }^{5)}$, \\ Tae TOMIYAMA ${ }^{1)}$, Yosuke SUGIURA ${ }^{1)}$, Nobutsune ICHIHARA ${ }^{1)}$ and Masao ASARI ${ }^{1)}$
}

${ }^{1)}$ First Department of Anatomy, School of Veterinary Medicine, Azabu University, 1-17-71, Fuchinobe, Sagamihara, Kanagawa 229-8501, ${ }^{2)}$ Laboratory of Physical Anthropology, Graduate School of Science, Kyoto University, Kitashirakawa-oiwakecho, Sakyo-ku, Kyoto 606-8502, ${ }^{3)}$ The University Museum, The University of Tokyo, 7-3-1, Hongo, Bunkyo-ku, Tokyo 113-0033, ${ }^{4)}$ Ueno Zoological Gardens, Tokyo 1108711 and ${ }^{5)}$ Department of Zoology, National Museum of Nature and Science, 3-23-1, Hyakunin-cho, Shinjuku-ku, Tokyo 169-0073, Japan

(Received 27 December 2008/Accepted 26 January 2009)

ABSTRACT. We dissected the hindlimb of a female western lowland gorilla and determined the muscle dimensions (mass, fascicle length, and physiological cross-sectional area: PCSA). Comparisons of the muscle parameters of the measured gorilla with corresponding reported human data demonstrated that the triceps surae muscles were larger and had more capacity to generate force than the other muscle groups in both species, but this tendency was more prominent in the human, probably as an adaptation to strong toe-off during bipedal walking. On the other hand, PCSAs of the extrinsic pedal digital flexors and digiti minimi muscles were larger in the western lowland gorilla, suggesting that the foot, particularly the fifth toe, has a relatively high grasping capability in the lowland gorilla.

KEY WORDS: foot, gorilla, muscle architecture.

J. Vet. Med. Sci. 71(6): 821-824, 2009

Gorillas are generally regarded as the most terrestrial of the extant hominoids. However, the degree of arboreality is known to vary among subspecies. Mountain gorillas $(G$. gorilla beringei) living in eastern central Africa seem to be the least arboreal, and the amount of time they spend above ground is only $7 \%$ in females and $2 \%$ in males [1]. On the other hand, western lowland gorillas ( $G$. g. gorilla) in western central Africa are observed to be more arboreal and are frequently found in trees higher than $20 \mathrm{~m}[1,9]$. Such differences in the degree of arboreality among the subspecies are correlated with foot anatomy. The foot of the western lowland gorilla has a relatively longer free portion of the first toe capable of opposing to the other four toes for grasping, whereas that of the mountain gorilla is relatively more humanlike and adapted for terrestrial locomotion [10,11].
Therefore, understanding subspecies variations in the muscular characteristics of the gorilla foot is important for interpreting functional adaptations of the foot in hominoids. However, although Payne et al. [8] have reported the muscle architecture of the gorilla's hindlimb, no studies so far have provided complete quantitative data on all of the foot muscles, including the intrinsic muscles.

In this study, we dissected the left hindlimb of a female western lowland gorilla to provide complete quantitative

* Correspondence to: Oishi, M., First Department of Anatomy, School of Veterinary Medicine, Azabu University, 1-17-71, Fuchinobe, Sagamihara, Kanagawa 229-8501, Japan.

e-mail:dv0502@azabu-u.ac.jp

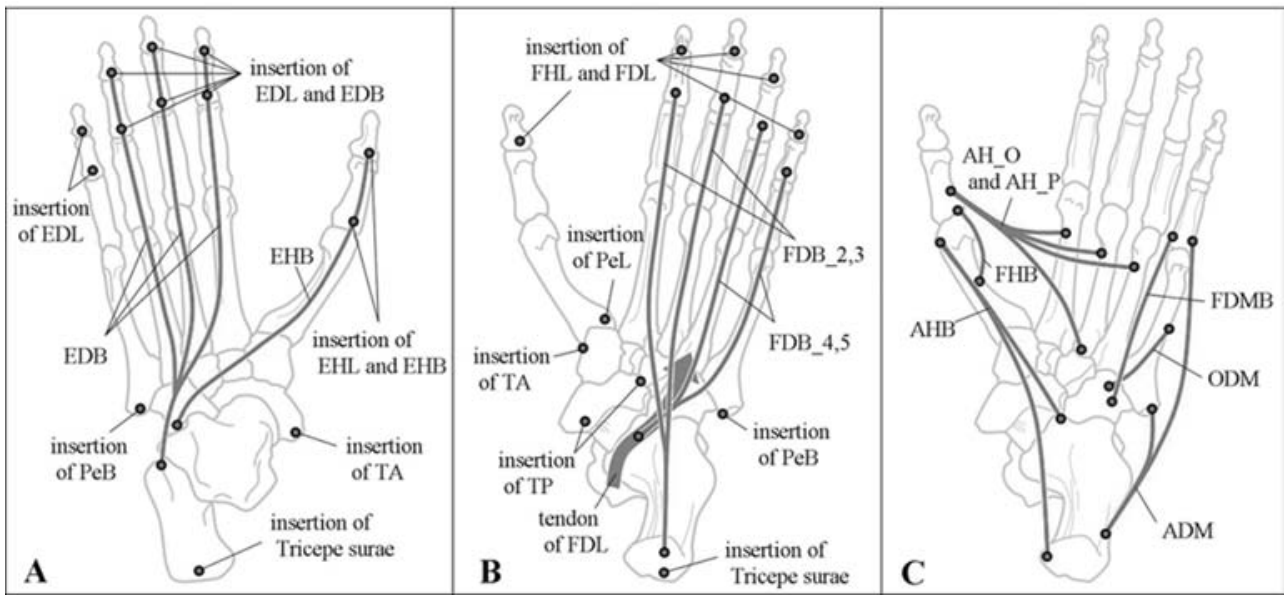

Fig. 1. Schematic diagram of the foot muscles of the lowland gorilla in dorsal (A) and planter (B, C) views The ankle dorsiflexor (TA, EHL, EDL), extrinsic digital flexor (FHL, FDL) and digital minimi muscles (ADM, FDMB, ODM, FDB_5) were well-developed in the lowland gorilla. Mm. lumbricals and $\mathrm{Mm}$. interossei are not included. Muscle name abbreviations are as detailed in Tables 1 and 2. 
Table 1. Foot and shank muscle dimensions in the lowland gorilla

\begin{tabular}{|c|c|c|c|c|}
\hline & Abbrevition & Mass (g) & $\operatorname{PCSA}\left(\mathrm{cm}^{2}\right)$ & $\mathrm{FL}(\mathrm{cm})$ \\
\hline Gastrocnemius and Plantaris & $\mathrm{Gas}+\mathrm{Pla}$ & 88.3 & 11.80 & 7.1 \\
\hline Soleus & Sol & 102.3 & 21.98 & 4.4 \\
\hline Popliteus & Pop & 4.9 & 0.93 & 5.0 \\
\hline Flexor hallucis longus & FHL & 58.1 & 7.42 & 7.4 \\
\hline Flexor digitorum longus & FDL & 22.1 & 2.67 & 7.8 \\
\hline Tibialis posterior & $\mathrm{TP}$ & 19.4 & 4.16 & 4.4 \\
\hline Tibialis anterior & TA & 58.2 & 7.16 & 7.7 \\
\hline Extensor hallucis longus & EHL & 10.9 & 1.12 & 9.2 \\
\hline Extensor digitorum longus_2,3 & EDL_2,3 & 9.4 & 0.97 & 9.1 \\
\hline Extensor digitorum longus_4 & EDL_ 4 & 7.6 & 0.84 & 8.5 \\
\hline Extensor digitorum longus_5 & EDL_5 & 13.5 & 1.35 & 9.4 \\
\hline Peroneus longus & PeL & 44.0 & 7.40 & 5.6 \\
\hline Peroneus brevis & $\mathrm{PeB}$ & 21.6 & 4.02 & 5.1 \\
\hline Peroneus tertius & PeT & 7.6 & 0.96 & 7.5 \\
\hline Extensor hallucis brevis & EHB & 4.7 & 1.29 & 3.4 \\
\hline Extensor digitotrum brevis_2 & EDB_2 & 4.0 & 0.63 & 6.1 \\
\hline Extensor digitotrum brevis_3 & EDB_3 & 2.9 & 0.65 & 4.2 \\
\hline Extensor digitotrum brevis_4 & EDB_4 & 3.0 & 0.73 & 3.8 \\
\hline Flexor digitorum brevis_2 & FDB_2 & 4.8 & 0.99 & 4.6 \\
\hline Flexor digitorum brevis_3 & FDB_3 & 6.3 & 0.98 & 6.0 \\
\hline Flexor digitorum brevis_4 & FDB_4 & 1.4 & 0.75 & 1.8 \\
\hline Flexor digitorum brevis_5 & FDB_5 & 0.7 & 0.48 & 1.4 \\
\hline Abductor hallucis brevis & AHB & 20.7 & 4.93 & 4.0 \\
\hline Flexor hallucis brevis & FHB & 10.9 & 5.31 & 1.9 \\
\hline Adductor hallucis, caput obliquum & AH_O & 7.8 & 2.53 & 2.9 \\
\hline Adductor hallucis, caput transversum & AH_T & 12.5 & 4.19 & 2.8 \\
\hline Abductor digiti minimi & ADM & 15.6 & 8.98 & 1.6 \\
\hline Flexor digiti minimi brevis & FDMB & 3.7 & 2.26 & 1.5 \\
\hline Opponens digiti minimi & ODM & 0.8 & 0.52 & 1.4 \\
\hline Dorsal interosseus_1 & DI_1 & 3.8 & 1.99 & 1.8 \\
\hline Dorsal interosseus_2 & DI_2 & 2.3 & 1.91 & 1.1 \\
\hline Dorsal interosseus_3 & DI_3 & 3.5 & 2.15 & 1.5 \\
\hline Dorsal interosseus_4 & DI_4 & 4.3 & 3.26 & 1.3 \\
\hline Plantar interosseus_1 & PI_1 & 3.2 & 2.60 & 1.1 \\
\hline Plantar interosseus_2 & PI_2 & 1.6 & 0.73 & 2.1 \\
\hline Plantar interosseus_3 & PI_3 & 1.5 & 1.08 & 1.3 \\
\hline Lumbricalis_1 & Lu_1 & 0.4 & 0.10 & 4.0 \\
\hline Lumbricalis_2 & Lu_2 & 0.9 & 0.21 & 4.1 \\
\hline Lumbricalis_3 & Lu_3 & 0.7 & 0.16 & 4.3 \\
\hline Lumbricalis_4 & Lu_4 & 0.6 & 0.14 & 4.2 \\
\hline
\end{tabular}

PCSA $=$ physiological cross-sectional area. $\mathrm{FL}=$ fascicle length.

data on the gorilla foot musculature. The cadaver (Museum No.: NSMT-M35965) was obtained from Ueno Zoological Gardens through the National Museum of Nature and Science, Tokyo, Japan. The gorilla died at the approximate age of 40 years, with a body mass of $69 \mathrm{~kg}$ at the time of death. The specimen was stored frozen until dissection.

A schematic diagram of the foot muscles in the lowland gorilla is provided in Fig. 1. The foot musculature of the lowland gorilla is essentially similar to that of the human and other great apes, although some structural variations are noted. For example, M. quadratus plantae, running from the calcaneus to the tendon of the extrinsic pedal digital flexor muscles, is known to occur in only $30 \%$ of the lowland gorilla [12]. This muscle was absent in the present specimen. Moreover, M. flexor digitorum brevis was separated into the superficial and deep heads in the present spec- imen as also observed in other great apes [10, 12]; the superficial head arose from the calcaneus and inserted to the second and third toes, and the deep head arose from the tendon of $M$. flexor digitorum longus and inserted to the fourth and fifth toes (Fig. 1B). However, this muscle consists of only the superficial head and sends its tendons to the lateral four toes in the human [4].

During dissection, the muscles listed in Table 1 were exposed and removed from the foot and hindlimb bones. Muscle mass was determined using an electronic balance. Each muscle belly was immersed in $10 \%$ formalin. Thereafter, the fascicle length was measured at three to six places using a caliper. The PCSA was calculated by dividing the muscle volume by the fascicle length. The muscle volume was obtained by dividing the muscle mass by the muscle density $\left(1.0597 \mathrm{~g} / \mathrm{cm}^{3}\right)$ [5]. We did not include the penna- 
Table 2. Comparisons of mass ratios and PCSA ratios in shank muscles with those of published gorilla (Gm and Gj) and human data

\begin{tabular}{|c|c|c|c|c|c|c|c|c|}
\hline & \multicolumn{4}{|c|}{ Mass ratios } & \multicolumn{4}{|c|}{ PCSA ratios } \\
\hline & This study & $\mathrm{Gm}$ & $\mathrm{Gj}$ & Human (SD) & This study & $\mathrm{Gm}$ & $\mathrm{Gj}$ & Human (SD) \\
\hline Triceps surae & 43.2 & 42.5 & 40.9 & $54.3(3.55)$ & 49.8 & 39.7 & 39.6 & $61.7(6.84)$ \\
\hline Extrinsic pedal digital flexors & 18.2 & 19.2 & 17.0 & $7.7(1.47)$ & 14.9 & 18.9 & 15.8 & $8.5(1.89)$ \\
\hline Dorsiflexors & 22.6 & 23.7 & 21.4 & $17.5(2.74)$ & 16.9 & 27.9 & 17.2 & $10.4(1.88)$ \\
\hline Other muscles & 16.1 & 14.6 & 20.7 & $20.4(1.16)$ & 18.4 & 13.6 & 27.4 & $19.4(4.11)$ \\
\hline
\end{tabular}

$\mathrm{Gm}$ and $\mathrm{Gj}$ are cited from reported gorilla data [8]. The two values for human are given as the means and standard deviations (SD) of data from two published studies [2,13]. Mass and PCSA ratios were calculated as subtotal of the constituent muscles of muscle groups. Muscle name abbreviations are as detailed in Table 1. Muscle groups: Triceps surae (Gas+Pla, Sol), Extrinsic pedal digital flexors (FHL, FDL), Dorsiflexors (TA, EHL, EDL), Other muscles (TP, PeL, PeT). M. popliteus and M. peroneus brevis are not included in other muscle group because complete data for the gorilla [8] and the human [2].

Table 3. Comparisons of mass ratios and PCSA ratios in foot muscles with those of published human data

\begin{tabular}{lcccccc}
\hline & \multicolumn{2}{c}{ Mass ratios } & & \multicolumn{2}{c}{ PCSA ratios } \\
\cline { 2 - 3 } \cline { 5 - 6 } & Gorilla & Human & & Gorilla & Human \\
\hline Hallucal muscles & 46.2 & 40.1 & & 36.8 & 38.3 \\
Digiti minimi muscles & 16.9 & 14.6 & & 24.7 & 13.4 \\
Intrinsic pedal digital extensors & 8.1 & 2.9 & & 4.1 & 3.8 \\
Intrinsic pedal digital flexors & 10.2 & 20.7 & & 5.5 & 16.4 \\
Interossei & 16.4 & 19.3 & & 27.7 & 25.7 \\
Lumbricals & 2.2 & 2.4 & & 1.2 & 2.3 \\
\hline
\end{tabular}

Human data are cited from Kura et al. [4]. Mass and PCSA ratios were calculated as subtotal of the constituent muscles of muscle groups. Muscle name abbreviations are as detailed in Table 1. Muscle groups: Hallucal muscles (EHB, AHB, FHB, AH_O, AH_T), Digiti minimi muscles (ADM, FDMB, ODM, FDB_5), Intrinsic pedal digital extensors (EDB_2-4), Intrinsic pedal digital flexors (FDB_2-4, M. quadratus plantae), Interossei (DI_1-4, PI_13), Lumbricals (Lu_1-4).

tion angle in our calculation of PCSA, because, in the twodimensional muscle model, it was difficult to correctly measure the angle of the three-dimensional fascicle within a muscle. For comparisons of muscle architecture, the mass and PCSA of each foot muscle were divided by the total foot muscle mass and PCSA to calculate mass and PCSA ratios, respectively. The mass and PCSA ratios of the shank muscles were calculated in the same manner.

To confirm the consistency of our measurements, the mass and PCSA ratios of the shank muscles were compared with those reported by Payne et al. [8] (two male western lowland gorillas, $\mathrm{Gm}$ and $\mathrm{Gj}$ ). Although a certain degree of variability exists, Table 2 indicates that the data acquired in the present study were generally in accordance with the previously reported data, suggesting that the described gorilla muscle architectural patterns appear to be reasonably representative.

Table 2 also shows compares the mass and PCSA ratios of gorilla and human shank musculature. The human data are taken from Wickiewicz et al. [13] and Friederich and Brand [2]. In both species, the triceps surae muscles were larger and had more capacity to generate force than the other muscle groups, but this tendency was found to be more prominent in the human, probably as an adaptation to strong toe-off during bipedal walking. On the other hand, the mass and PCSA ratios of the ankle dorsiflexor and extrinsic digi- tal flexor muscles were larger in the gorilla (Table 2).

With respect to the intrinsic foot muscles (Table 3), the digital flexor muscles were relatively smaller in the gorilla because $M$. quadratus plantae is relatively large in the human foot [4], whereas this muscle is rudimentary in the gorilla's foot $[3,12]$. Instead, the PCSA ratio of the digital minimi muscles was relatively larger in the gorilla, possibly indicating that the lowland gorilla has a greater grasping capability of the fifth toe (Table 3).

This study is the first complete report on the muscular dimensions of the foot in the lowland gorilla. The mass, PCSA, and fascicle length of the thigh muscles from the same specimen are also presented in the Appendix. Such complete data of the muscle dimensions are essential for understanding the form-function relationship of musculature in hominoids [e.g., 6, 7]. We hope to have more opportunities to dissect the feet of both lowland and mountain gorillas for comparative analysis of the intra- and inter-subspecies variations in muscle architecture in gorillas, which might reflect differences in their habitats and locomotor behaviors.

ACKNOWLEDGEMENTS. The authors would like to thank all of the staff of Ueno Zoological Gardens (Tokyo, Japan) for kindly allowing us to dissect the specimen. This study was partly supported by the Cooperation Research 
Appendix. Thigh muscle dimensions in the lowland gorilla

\begin{tabular}{|c|c|c|c|}
\hline & $\operatorname{Mass}(\mathrm{g})$ & $\operatorname{PCSA}\left(\mathrm{cm}^{2}\right)$ & $\mathrm{FL}(\mathrm{cm})$ \\
\hline Psoas major and Psoas minor & 73.3 & 8.57 & 8.1 \\
\hline Iliacus & 79.8 & 11.58 & 6.5 \\
\hline Tensor fascia latae & 25.7 & 1.58 & 15.4 \\
\hline Gluteus maximus & 328.2 & 31.97 & 9.7 \\
\hline Gluteus medius & 391.0 & 36.04 & 10.2 \\
\hline Prifoermis & 37.5 & 6.07 & 5.8 \\
\hline Gluteus minimus & 57.3 & 8.99 & 6.0 \\
\hline Quadratus femoris & 10.5 & 2.24 & 4.4 \\
\hline Superior gemellus & 2.7 & 0.96 & 2.6 \\
\hline Inferior gemellus & 1.4 & 0.33 & 3.9 \\
\hline Biceps femoris, caput longum & 103.0 & 7.06 & 13.8 \\
\hline Biceps femoris, caput breve & 44.6 & 4.86 & 8.7 \\
\hline Semimembranosus & 19.4 & 1.25 & 14.6 \\
\hline Semitendinosus & 100.9 & 7.00 & 13.6 \\
\hline Gracilis & 91.1 & 3.40 & 25.3 \\
\hline Sartorius & 19.2 & 0.55 & 32.9 \\
\hline Adductor longus & 40.7 & 3.57 & 10.8 \\
\hline Adductor brevis & 51.8 & 7.70 & 6.4 \\
\hline Adductor magnus & 442.8 & 23.88 & 17.5 \\
\hline Pectineus & 15.1 & 2.01 & 7.1 \\
\hline Obturatorius internus & 35.3 & 6.61 & 5.0 \\
\hline Obturatorius externus & 37.2 & 5.69 & 6.2 \\
\hline Rectus femoris & 79.6 & 7.36 & 10.2 \\
\hline Vastus lateralis & 174.7 & 19.23 & 8.6 \\
\hline Vastus medialis & 105.3 & 12.95 & 7.7 \\
\hline Vastus intermedius & 117.8 & 14.07 & 7.9 \\
\hline
\end{tabular}

$\mathrm{PCSA}=$ physiological cross-sectional area. $\mathrm{FL}=$ fascicle length.

Program of Primate Research Institute, Kyoto University, by the JSPS core-to-core program HOPE, and by a Grant-inAid for Scientific Research on Priority Areas "Emergence of Adaptive Motor Function through Interaction between Body, Brain and Environment" from the Japanese Ministry of Education, Culture, Sports, Science and Technology.

\section{REFERENCES}

1. Doran, D. M. 1996. Comparative positional behavior of the African apes. pp 213-224. In: Great Ape Societies (McGrew, W. C., Marchant, L. F. and Nishida, T., eds.), Cambridge University Press, Cambridge.

2. Friederich, J. A. and Brand, R. A. 1990. Muscle fiber architecture in the human lower limb. J. Biomech. 23: 91-95.

3. Gregory, W. K., editor. 1950. The Anatomy of the Gorilla: the Henry Cushier Raven Memorial Volume. Columbia University Press, New York.

4. Kura, H., Luo, Z. P., Kitaoka, H. B. and An, K. N. 1997. Quantitative analysis of the intrinsic muscles of the foot. Anat. Rec. 249: 143-151.

5. Mendez, J. and Keys, A. 1960. Density and composition of mammalian muscle. Metabolism 9: 184-188.

6. Ogihara, N., Kunai, T. and Nakatsukasa, M. 2005. Muscle dimensions in the chimpanzee hand. Primates 46: 275-280.

7. Oishi, M., Ogihara, N., Endo, H. and Asari, M. 2008. Muscle architecture of the upper limb in the orangutan. Primates 49: 204-209.

8. Payne, R. C., Crompton, R. H., Isler, K., Savage, R. Vereecke, E. E., Günther, M. M., Thorpe, S. K. S. and D'Août, K. 2006. Morphological analysis of the hindlimb in apes and humans. I. Muscle architecture. J. Anat. 208: 709-724.

9. Remis, M. 1995. Effects of body size and social context on the arboreal activities of lowland gorillas in the Central African Republic. Am. J. Phys. Anthropol. 97: 413-433.

10. Sarmiento, E. E. 1994. Terrestrial traits in the hands and feet of gorillas. Am. Mus. Nat. Hist. Novitates 3091: 1-56.

11. Schultz, S. H. 1934. Some distinguishing characters of the mountain gorilla. J. Mammal. 12: 51-61.

12. Straus, W. L. Jr. 1930. The foot musculature of the highland gorilla (Gorilla beringei). Q. Rev. Biol. 5: 261-317.

13. Wickiewicz, T. L., Roy, R. R., Powell, P. L. and Edgerton, V. R. 1983. Muscle architecture of the human lower limb. Clin. Orthop. 179: 275-283. 